\title{
Change of Preferential Liquid Breakthrough Pathways in PEMFC Gas Diffusion Layers: Evidence of Pressure-Induced Dynamic Transport
}

\author{
S. Chevalier, C. Josset and B. Auvity \\ Laboratoire de Thermique et Énergie de Nantes (CNRS UMR 6607), Polytech Nantes, \\ University of Nantes, La Chantrerie, rue Christian Pauc, Nantes, France.
}

\begin{abstract}
In this work, the change of liquid water preferential pathways through PEM fuel cell Gas Diffusion Layers (GDL) with ex situ devices is investigated. A capillary network constituted of two connected micro channels is first developed. It is shown that once the larger channel has been invaded, the pressure variation due to the growth of the water droplets at the channel tip enables the liquid water to invade the smaller capillary channel. At the end, the liquid preferential path is modified. This result is then generalized considering a real GDL. Liquid water is injected through the GDL to a micro channel, and changes in liquid water pathways are observed after several hours of operations. The reported experimental observations show that pressure-induced dynamic transport due the water droplet eruption in the channel has to be taken into account to predict the preferential pathway after long time of fuel cell operation.
\end{abstract}

\section{Introduction}

The water management in PEM Fuel Cell is a key issue to get stable and high performances. Water is present in liquid form in the GDL and channel, especially at temperature below $70^{\circ} \mathrm{C}-80^{\circ} \mathrm{C}(1)$. Water produced in the catalyst layer may not enter the GDL in liquid form but may condensate due to existing temperature gradient through the GDL, $(2,3)$. Privileged condensation points may appear in the GDL under the rib (4) and water is evacuated in liquid form through the GDL to the GDL/channel interface (5).

Evidence of preferential pathways for liquid in GDL has been observed both in in situ studies (6) and ex situ studies (7). The existence of preferential pathways in porous media is well described by the Invasion-Percolation (IP) theory which has been introduced in computational code referred as Pore-Network modelling (8).

The transport of the liquid phase in GDL has been shown to be discontinuous (9). This dynamic phenomenon was previously observed in porous media and referred to Hayne's jump. For instance, interfacial jumps were observed in porous media (10). The preferential pathways inside GDL may vary in location over time both in in situ and ex situ conditions $(5,7)$. In (7), the authors hypothesized a scenario that may not explain the whole phenomena. Besides, they showed that the presence of a Micro Porous Layer (MPL) stabilizes the breakthrough location. In an ex situ set-up, changes in breakthrough location appear in (11) and were attributed to water impurities. 
The motivation of the present work is to clarify the mechanisms leading to change in preferential paths location when liquid is transported inside a porous media (here a GDL). In a recent study, the present authors studied a simple network composed of two connected capillaries to mimic two neighbouring liquid water pathways in porous media (12). They built a model taking into account only the friction effects along the capillary tubes and the capillary pressure effect at the capillary tips through the Laplace law, see figure 1. This study showed that the driving parameters to change from one preferred pathways (being the lager capillary, figure 1(a)) to oscillating state between the two possible pathways, see figure 1(b) are: the hydraulic diameter ratio between the two capillary tubes, a generalized capillary number taking into account the classical capillary number multiplied by the non-dimensionnal tube length and the detachment height of the droplets formed at the tube tips. In short, the transition from one preferred pathway to another is very likely to occur when viscous effects are no more negligible compared to capillary effects i.e. increasing the incoming liquid water flow rate and when the characteristic dimension of two neighboring capillary tubes is close to one. As in GDL there is a distribution of pore radius quite centered around an average value, this mechanism may be promoted. This study opens the door to a possible explanation as to why the liquid pathways may vary in porous media.

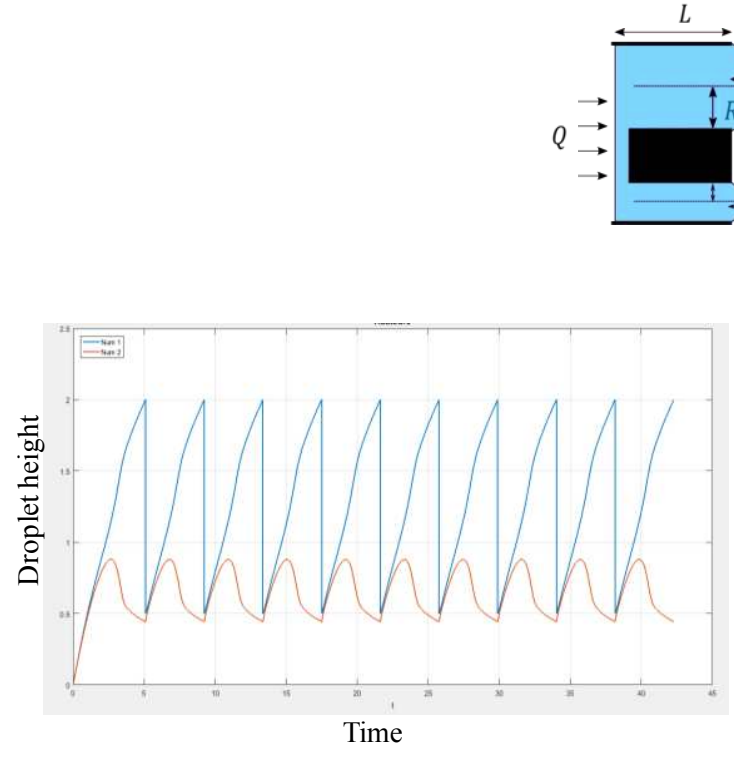

(a)

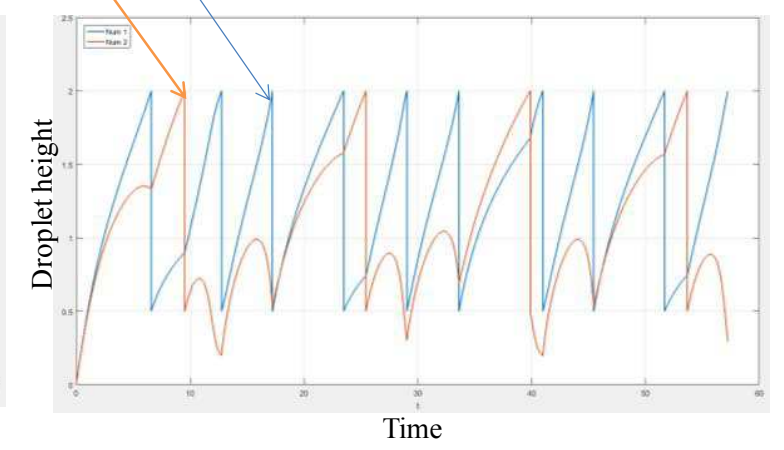

(b)

Figure 1. Temporal evolution of the droplet meniscus heights in the case of two connected capillaries as predicted by the model developed in (12). (a) stationary capillary regime : liquid water droplets are emitted only from the larger capillary. (b) oscillating viscous-capillary regime: liquid water droplets are emitted from both capillaries.

In the present paper, two ex situ micro fluidic devices are developed to further observe and analyze the preferential pathways location and their possible change. In the first ex situ device, a simple network of two connected micro channels is considered. With this setup, we shall see that the droplet emission from one channel induces meniscus motion in the other channel due to the pressure variations occurring while the droplets are formed and ejected. In the second ex-situ device, a GDL is molded inside a PDMS block and liquid water is injected underneath. A gas channel is placed on top of the GDL to reproduce the conditions encountered in PEM fuel cell. Experiments are run for extended 
period of time. A change in preferential breakthrough locations is observed even in presence of a MPL and purified water. As we intend to address the problem of liquid invasion in porous media, all experiments are conducted at room temperature with liquid water to limit the evaporation phenomenon.

\section{First Ex Situ Device: Two Connected Micro Channels}

We first consider the experimental device sketched on figure 2. A network of two channels is machined inside a Teflon block. Teflon material is privileged as the water is non-wetting with Teflon (hydrophobic condition). The Teflon block is covered with a transparent PDMS block for optical access. In terms of wettability, PDMS is neutral with liquid water (contact angle of about $90^{\circ}$ ). The main channel through which the water is injected is a straight channel with a hydraulic diameter of 320 micrometers and $6 \mathrm{~cm}$ long. The channel cross section is square. A $45^{\circ}$ junction with a smaller channel (300 micrometers of hydraulic diameter) is realized. This channel enlarges to become a 500 micrometers hydraulic diameter channel. At the beginning of the experiment, the channels network is free of liquid water and filled with air. This channel arrangement has been designed so that in capillary dominated flow regime with non-wetting conditions, the liquid invasion appears only in the main straight channel and a meniscus in the smaller channel gets formed at the junction. This is well predicted by the IP theory. We intend to observe what happens when the injected flow rate is increased and thus when the oscillating viscous-capillary state revealed in the figure 1(b) is promoted. In that sense, the ratio of hydraulic diameter between the main channel and the smaller one has been chosen equal to 1.07 , a value close to one for which the transition from stationary state (figure 1(a)) and oscillating state(figure 1(b)) appears at lower capillary number (12).

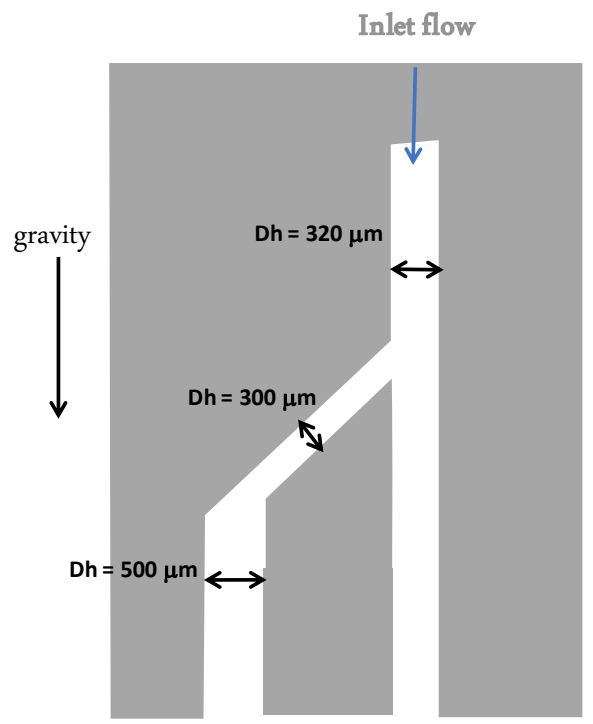

Figure 2. Sketch of the first ex situ device: values of the hydraulic diameters for the three portions of channels are presented. The channels are machined in a Teflon block (represented in gray on the figure). A PDMS transparent block is placed on top of the Teflon block to close the channel.

The inlet water flow rate is set to $50 \mathrm{ml} / \mathrm{min}$ which correspond to a capillary number of 1.6 $10^{-4}$. The liquid water is injected thanks to a syringe pump (Legato $110, \mathrm{KD}$ Scientific). Water is colored using fluorescein. Images are recorded using a camera (Chameleon CMLN-1352C, Edmund Optics Ltd) with an acquisition rate of 1 frame/sec. 
Close to the channel exit, air is blown with a slow flow rate adjusted to limit the size of the droplets at the channel tip. Actually, to promote the transition from the stationary to the oscillating state, it has been shown in (12) that the droplet height has to be limited. Hence, in the experiment, with the air stream, the droplet gets detached from the device before reaching the size at which gravity would naturally detach it.

Figure 3 presents images taken during the experiment conducted in the conditions presented above. $t_{0}$ corresponds to the time at which the water appears at the injection point . After 40s, see figure 3(a), the liquid front passed the junction and as predicted by the IP theory, water invades only the larger channel. At $t_{0}+1 \mathrm{~min}$, few droplets have already been formed at the larger channel exit tip and it can be observed that the meniscus in the smaller channel, initially close to the junction, moved forward. This is more evident on figure $3(\mathrm{c})$, at $\mathrm{t}_{0}+6 \mathrm{~min}$, after the formation and ejection of many droplets. During each droplet formation and emission, pressure builds up in the liquid domain as it increases in the droplet due to Laplace law. This pressure gets high enough to push and displace the meniscus located along the smaller channel. Once the meniscus reaches the enlargement of the cross section, see figure 3(d), liquid flow appears only in this portion of the network and no more droplets are emitted from the main channel exit. Actually, the meniscus positioned at the main channel exit inflates and deflates as droplets are emitted from the other channel exit but the hydraulic diameter ratio has been chosen large enough $(500 / 300=1.67)$ to not permit the oscillating state to appear in this flow condition. Then, a situation equivalent to figure 1(a) is obtained.

From this experiment, it is shown that the preferential breakthrough path can be modified in a capillary network due to the eruptive transient dynamic of water droplet emission. Indeed, during a first period of the experiment, the breakthrough point is located at the tip of the main channel. The situation changes to switch to the other channel exit due to the progressive moving of the meniscus inside the network. This dynamic behavior is not taken into account into the static IP theory. Moreover, not only the breakthrough location has changed but also the liquid saturation in the network. Comparing images figure 3(b) and 3(d), the network liquid saturation has increased.

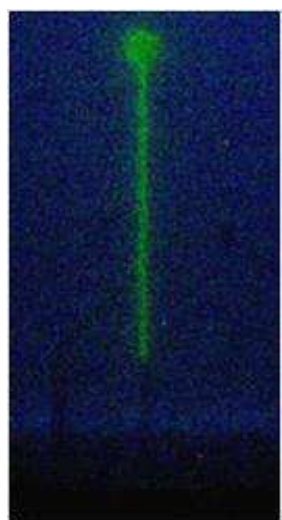

(a)

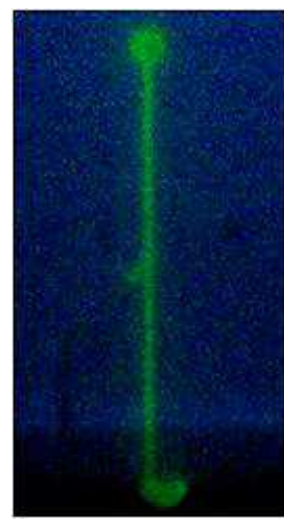

(b)

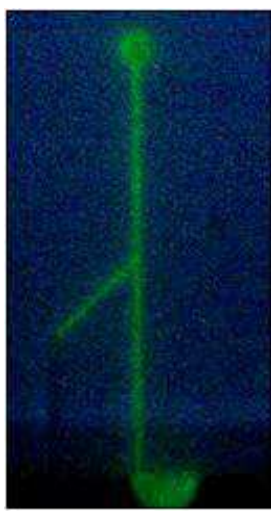

(c)

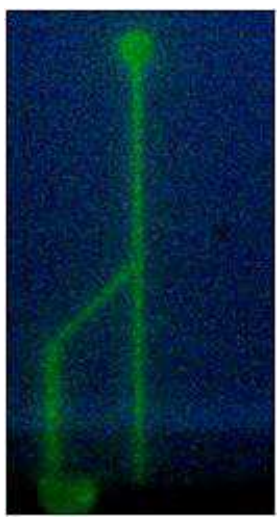

(d)

Figure 3. Flow visualization with device presented figure 2: (a) $t_{0}+40 \mathrm{~s}$; (b) $t_{0}+1 \mathrm{~min}$; (c) $\mathrm{t}_{0}+6$ min; (d) $\mathrm{t}_{0}+8 \mathrm{~min}$. 


\section{Second Ex Situ Device: Embedded GDL in PDMS Device}

We now consider a second ex-situ device with a commercial GDL (SGL 35 BC). In this experiment, liquid water is injected in a GDL compared to the simple network considered in the previous section. The motivation for this experiment is to observe the dynamics of breakthrough location changes. Actually, as recalled in the introduction section, changes in breakthrough locations for GDL have already been observed. Though, the idea of the present experiment is a first attempt to qualify the dynamics of the phenomenon in conditions close to these encountered in PEM fuel cell. Thus, on one hand, in the experiment, a gas stream is imposed on the top the GDL as liquid water is injected underneath. On the second hand, we shall see that very long operation time is needed to observe breakthrough location changes ( $5 \mathrm{~h}$ of experiments to see 3-4 location changes).

Figure 4(a) presents a schematic of the set-up. The GDL sample is molded in a PDMS block. This design has been chosen as leakages are very difficult to prevent in such material assembly. Indeed, the sealing around the GDL needs to be very good, otherwise injected water or gas may flow in parallel parasitic paths (13). Figure 4(b) shows pictures of the setup with the three constituting parts. Air channel is $8 \mathrm{~cm}$ long and has a square cross section of $1 \mathrm{~mm}^{2}$. The embedded GDL is $8 \mathrm{~cm}$ long and $1 \mathrm{~cm}$ width. Pressure is measured in the water cavity underneath the GDL and the pressure drop along the gas channel is measured as well.

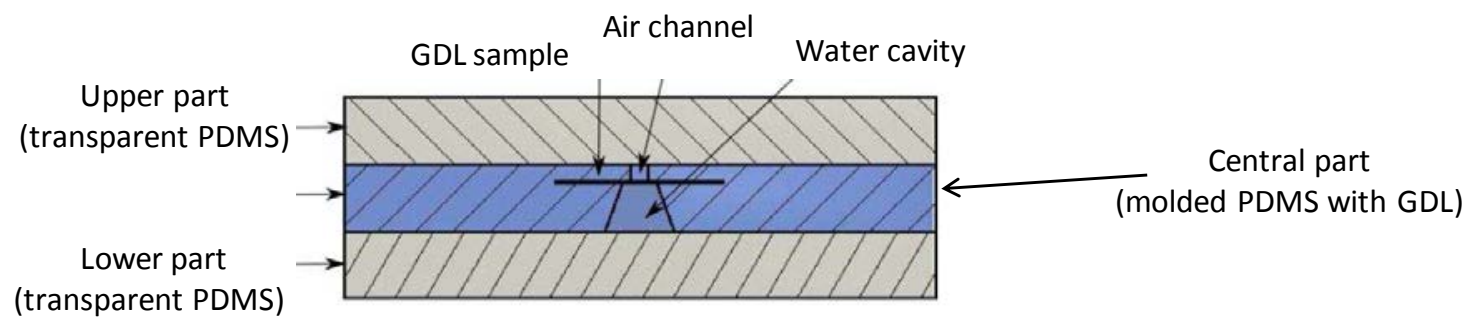

(a)

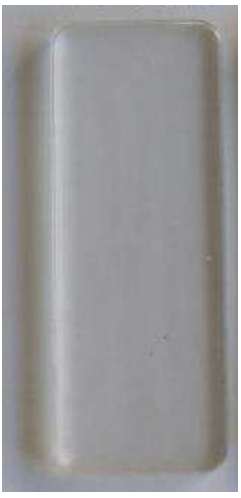

Upper part

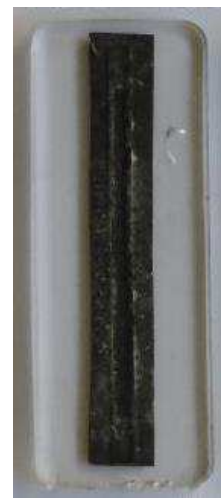

Central part

(b)

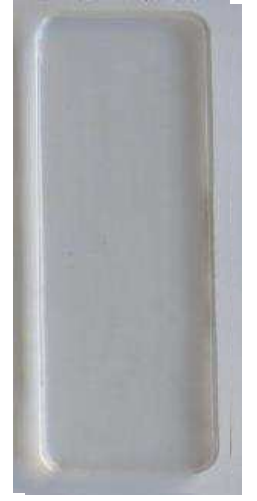

Lower part

Figure 4. (a) Schematic of the GDL embedded setup (b) Pictures of the three constituting parts.

Water and gas flow rates are chosen to reproduce PEM fuel cell conditions, refer to (14) where they are discussed. The experiment presented in this section is conducted with a water volume flow rate of $10.5 \mu \mathrm{l} / \mathrm{min}$ and a gas flow rate of $43 \mathrm{~cm}^{3} / \mathrm{min}$. Considering an average pore diameter of $30 \mu \mathrm{m}$ we can estimate a Capillary number the order of 5.10- 
4 in the GDL. This value is in the same range as the one considered in the previous section.

Figure 5 shows the simultaneous measurements of pressure in the water cavity, 5(a) and in the gas channel 5(b) as well as some images of the liquid flow in the gas channel $5(\mathrm{c})$.
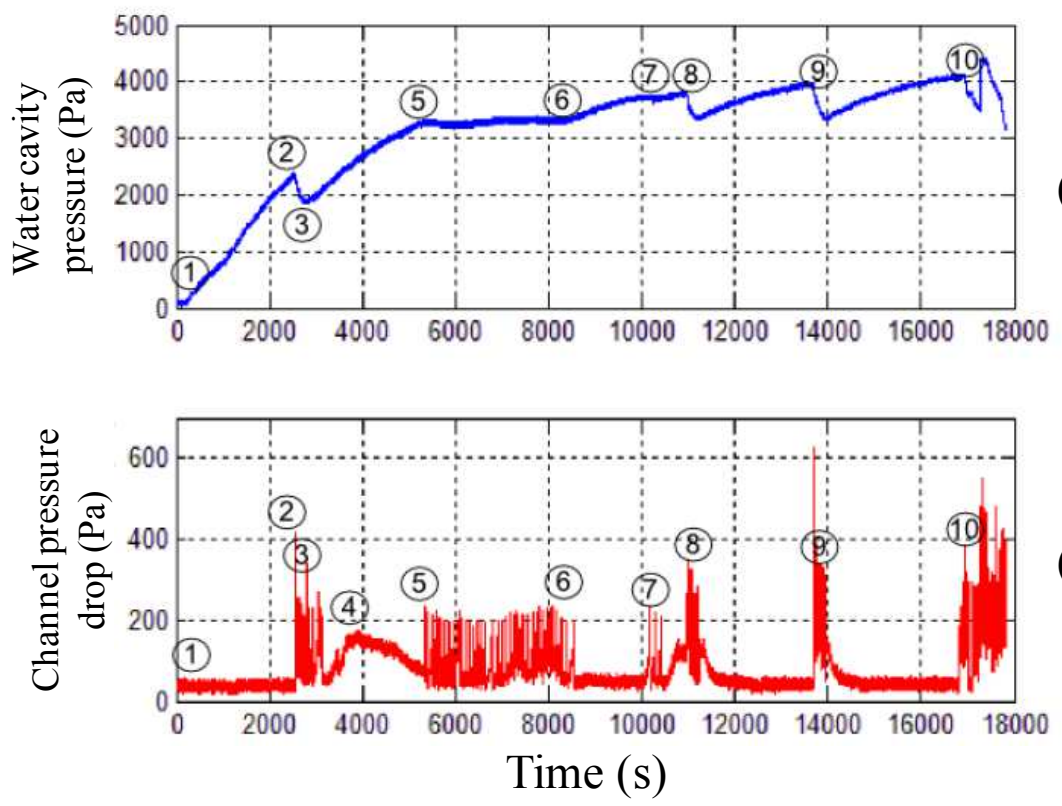

(b)

(2)

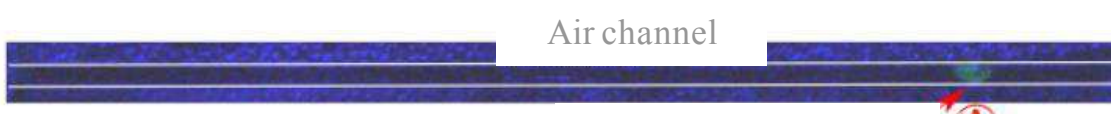

Air flow

(B)

Breakthrough locations

\section{$\longleftarrow$}

(3)

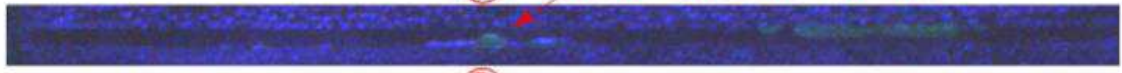

(B)

(4)

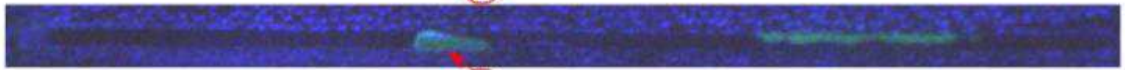

(B)

(5)

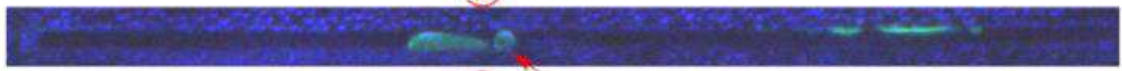

(B)

(7)

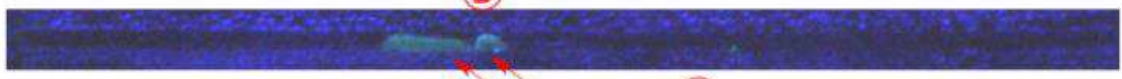

(C)

(8)

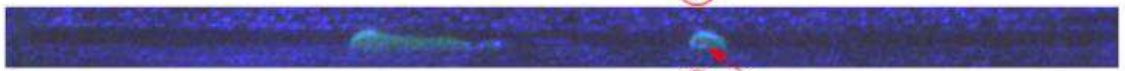

(C)

(9)

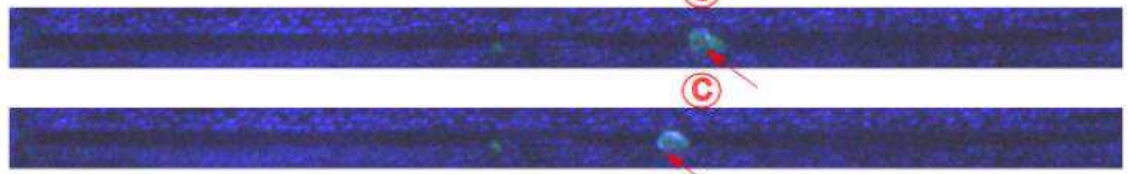

(c)

Figure 5. (a) Pressure signal in the water cavity (b) Pressure signal in the air channel (c) Breakthrough locations corresponding to the specific times of the figure (a) and (b).

Below is a short description of the events occurring during the experiment:

- From 1 to 2, liquid water is injected. No liquid droplet is emitted from the GDL in the gas channel. The pressure in the channel is constant. The pressure elevation in the 
water cavity is sometimes referred as "the system expansion" (15). It increases till it reaches the breakthrough value.

- From 2 to 3, liquid droplets are emitted either from breakthrough location A or B, figure 5(c). Emissions of droplets are detected from the pressure drop signal as spikes. The formation of liquid droplet in the channel reduces the cross section and hence increases the pressure drop. Once the droplet is expelled from the gas channel, the pressure drop falls down. During this first series of droplets emission, the pressure in the water region decreases. This may be interpreted as a liquid discharge from the GDL.

- From 3 to 5, no droplet breaks through the GDL. It lasts for about 40 minutes. During this time, the liquid pressure builds up inside the GDL. A pressure drop "bump" may be observed on image 4 figure 5(c). This is due to the presence of a long droplet stuck along the channel at the location B. This droplet slowly disappears from 4 to 5 .

- From 5 to 6 a sequence almost similar to what happens from 2 to 3 is observed: regular emissions of liquid droplets revealed both on images and on the pressure drop signal. Two differences though between both sequences: the breakthrough location is only at B and the water cavity pressure stays constant. This sequence lasts for 50 minutes.

- From 6 to 7, no droplet emission and slow water pressure build up.

- From 8, for few minutes (and also from 9 for few minutes), regular droplets emissions at location $\mathrm{C}$.

\section{Discussion}

Observations made on both ex situ devices may be conciliate in order to give a possible explanation on the physics leading to breakthrough location changes and possible liquid saturation modification during liquid invasion.

During the sequences of liquid droplets emissions at the GDL/channel interface, figure 5 , pressure in the liquid domain in the GDL strongly varies due to the formation and ejection droplet process. The pressure variation induces motions of the numerous menisci present in the capillary fingering network in the GDL. The meniscus motion observed in the smaller channel on figure 3 is a nice observation of this phenomenon. The menisci motions potentially modify the liquid saturation state inside the GDL, acting on the pressure in the water liquid domain as observed during sequences 2-3, 5-6 in figure 5 . A new liquid water pathway may then be found inside the GDL as the one occurring between figure 3(c) and 3(d). A significant period of time is however needed for the liquid water to reach again the GDL/channel interface. During this period no droplet is emitted. Once a new breakthrough point is reached, a series of droplets emissions may then be initiated.

\section{Conclusion}

With two ex situ experimental set-ups, we observe that the eruptive nature of droplet emissions from a GDL into a gas channel promotes important pressure variation in the liquid domain inside the GDL. This may lead to a redistribution of liquid water inside the 
GDL and then change the liquid breakthrough location. This result brings new insight into the physics of liquid invasion in thin porous media where boundary effects (here GDL/channel interface) are dominant. In this case, the well established Invasion Percolation theory is not sufficient for the prediction of liquid saturation for long operation time.

\section{Acknowledgments}

The authors would like to gratefully acknowledge Mr. G. Biotteau and C. Le Bozec for their technical assistance in the design and realization of the experimental setup. The research leading to these results has received funding from the People Programme (Marie Curie Actions) of the European Union's Seventh Framework Programme (FP7/20072013) under REA grant agreement $n^{\circ}$ PCOFUND-GA-2013-609102, through the PRESTIGE programme coordinated by Campus France.

\section{References}

1. TJ Dursch, MA Ciontea, GJ Trigub, CJ Radke, AZ Weber, ECS Trans. 50 (2) (2013)

2. A Thomas, G Maranzana, S Didierjean, J Dillet, O Lottin, J. Electrochem. Soc. 160 (2) (2013)

3. Hasegawa, T., Imanishi, H., Nada, M., and Ikogi, Y., SAE Technical Paper 201601-1185 (2016)

4. B. Straubhaar, J. Pauchet and M. Prat, Int. J. Hydrogen Energy, 40 (35) (2015)

5. I. Manke, C. Hartnig,N. Kardjilov, H. Riesemeier, J.Goebbels, R. Kuhn, P. Krüger, J. Banhart, Fuel Cells, 10 (1) (2010)

6. Tüber K., Pocza D. and Hebling C. Journal of Power Sources, 124 (2) (2003)

7. Z. Lu, M.M. Daino, C. Rath, S.G. Kandlikar Int. J. Hydrogen Energy 35 (9) (2010)

8. E.F. Medici and J.S. Allen ECS Trans. 41(1) (2011)

9. I. Manke, C. Hartnig, M. Grünerbel, W. Lehnert, N. Kardjilov, a. Haibel, a. Hilger, J. Banhart, and H. Riesemeier Appl. Phys. Lett., 90(17) (2007)

10. F. Moebius and D. Or J. Colloid Interface Sci. 377(1) 2012.

11. C. Quesnel, R. Cao, J. Lehr, A.M. Kietzig, A. Z. Weber and J.T. Gostick J. Phys. Chem. C 119 (2015)

12. S. Chevalier, C. Josset and B. Auvity Physics of Fluids, 29, 102102 (2017)

13. B. Auvity, G. Giacoppo, G. Squadrito and E. Passalacqua International Conference on Hydrogen Energy, 9-11 May 2010, Hammamet, Tunisie.

14. G. Flipo, C. Josset, J. Bellettre and B. Auvity Int. J. Hydrogen Energy, 41(34), 2016.

15. A. Bazylak, D. Sinton, and N. Djilali J. Power Sources, 176(1) (2008) 